\title{
Thermal Error Compensation of the Wear-Depth Real-Time Detecting of Self-Lubricating Spherical Plain Bearings
}

\author{
Zhan-Qi Hu*, Wei Li, Yu-Lin Yang, Bing-Li Fan and Hai-Li Zhou
}

\begin{abstract}
The spherical plain bearing test bench is a necessary detecting equipment in the research process of self-lubricating spherical plain bearings. The varying environmental temperatures cause the thermal deformation of the wear-depth detecting system of bearing test benches and then affect the accuracy of the wear-depth detecting data. However, few researches about the spherical plain bearing test benches can be found with the implementation of the detecting error compensation. Based on the self-made modular spherical plain bearing test bench, two main causes of thermal errors, the friction heat of bearings and the environmental temperature variation, are analysed. The thermal errors caused by the friction heat of bearings are calculated, and the thermal deformation of the wear-depth detecting system caused by the varying environmental temperatures is detected. In view of the above results, the environmental temperature variation is the main cause of the two error factors. When the environmental temperatures rise is $10.3^{\circ} \mathrm{C}$, the thermal deformation is approximately $0.01 \mathrm{~mm}$. In addition, the comprehensive compensating model of the thermal error of the wear-depth detecting system is built by multiple linear regression (MLR) and time series analysis. Compared with the detecting data of the thermal errors, the comprehensive compensating model has higher fitting precision, and the maximum residual is only $1 \mu \mathrm{m}$. A comprehensive compensating model of the thermal error of the wear-depth detecting system is proposed, which provides a theoretical basis for the improvement of the real-time wear-depth detecting precision of the spherical plain bearing test bench.
\end{abstract}

Keywords: Self-lubricating spherical plain bearing, Wear depth, Bearing test bench, Thermal error, Error compensation

\section{Introduction}

The self-lubricating spherical plain bearings are special journal bearings which inlay or bond the self-lubricating solid materials between the inner and outer races. These bearings have some special characteristics, such as maintenance-free, compact structure, and low coefficient of friction. Therefore, the self-lubricating spherical plain bearings are widely applied to aviation and aerospace fields [1].

In the research process of self-lubricating spherical plain bearings, the bearing performance and its life

*Correspondence: Ronghu118@163.com

Aviation Key Laboratory of Science and Technology on Generic

Technology of Aviation Self-Lubricating Spherical Plain Bearing, Yanshan University, Qinhuangdao 066004, China evaluation are fundamental because they provide guarantees for analyzing the tribological mechanism, determine the rated dynamic or static loading and forecast the bearing life. And the spherical plain bearing test bench is a necessary detecting equipment in the performance and life evaluation. Meanwhile, three performance parameters of spherical plain bearings (the wear depth, the friction torque and the friction temperature) need to be detected in real time. Particularly, the wear depth is the most important parameter for the tribological properties and the life of self-lubricating spherical plain bearings.

However, due to various error factors, such as temperature variations, loading changes, electromagnetic interference, the wear of assistant rolling bearings, etc. which affect the real-time wear-depth detecting precision of the spherical plain bearing test bench, 
the evaluating accuracy of the tribological property of spherical plain bearings is reduced. Among above factors, the thermal errors caused by the temperature variation are the main factors [2]. How to reduce the thermal errors of the bearing test bench is important for the evaluation of self-lubricating spherical plain bearings.

At present, the thermal error compensation has been intensively studied in precise CNC machine [3], yet few researches in the spherical plain bearing test benches. The existing researches only build the thermal error models, and do not compensate effectively the thermal errors. Zhou [4] built the thermal error compensation model of the spherical plain bearing test bench under the high temperature operating condition, but did not verify the fitting precision and the applicability of this model, nor compensated the thermal errors. Hu et al. [5] made a comprehensive compensating model for the wear-depth detecting system of the spherical plain bearing test bench based on the multi-body kinematics and verified its accuracy by FEM, but didn't compensate the thermal errors, either. Li et al. [6], designed a wear-depth detecting module with a mechanical compensating device, which can online compensate the thermal errors caused by varying environmental temperatures, but still not verified the compensation efficiency of this method in the experiment. Relevant researches of CNC machine can be used on thermal compensating method, bringing in software-based compensating error method. In other words, on the basis of the thermal deformation analysis, the mathematical compensating model of the thermal errors is built, and then the thermal deformation is predicted in the control system. At last, the thermal errors are compensated in real time $[7,8]$. In this process, many mathematical methods are used for the thermal error modeling, such as, regression analysis method [9], FEM, neural networks [10], time series analysis [11], screw theory, fuzzy theory [12], grey theory [13], support vector machine [14], genetic algorithm [15], ant colony algorithm [16].

Based on the self-made modular spherical plain bearing test bench, this paper calculates the thermal errors caused by the friction heat of the spherical plain bearing and the assistant rolling bearing, and detects the thermal deformation of the detecting system caused by the environmental temperature variation. According to the above experimental data, the comprehensive compensating model of the thermal error of the weardepth detecting system is built by MLR and time series analysis, and the applicability of the comprehensive compensating model is verified in the experiment.

\section{Wear-Depth Detecting Principle of Spherical Plain Bearings}

The self-made test bench of self-lubricating spherical plain bearings is composed of six modules, namely, the transmission module, the hydraulic loading module, the bearing fixture module, the wear-depth detecting module, the environmental simulation module and the control module [17]. The schematic diagram and the photograph of the bearing test bench are shown in Figure 1, and the schematic diagram of the wear-depth detecting system (the combination of the bearing fixture module and the wear depth detecting module) is shown in Figure 2. In the bearing fixture module, the spherical plain bearing is put into the T-type plate, and the testing spindle passes through the bearing inner ring. In wear-depth detecting module, the top of the measuring sleeve is fixed on the base of the test bench, and the contacting clamp of the displacement sensor is fastened on the bottom of the measuring sleeve, so

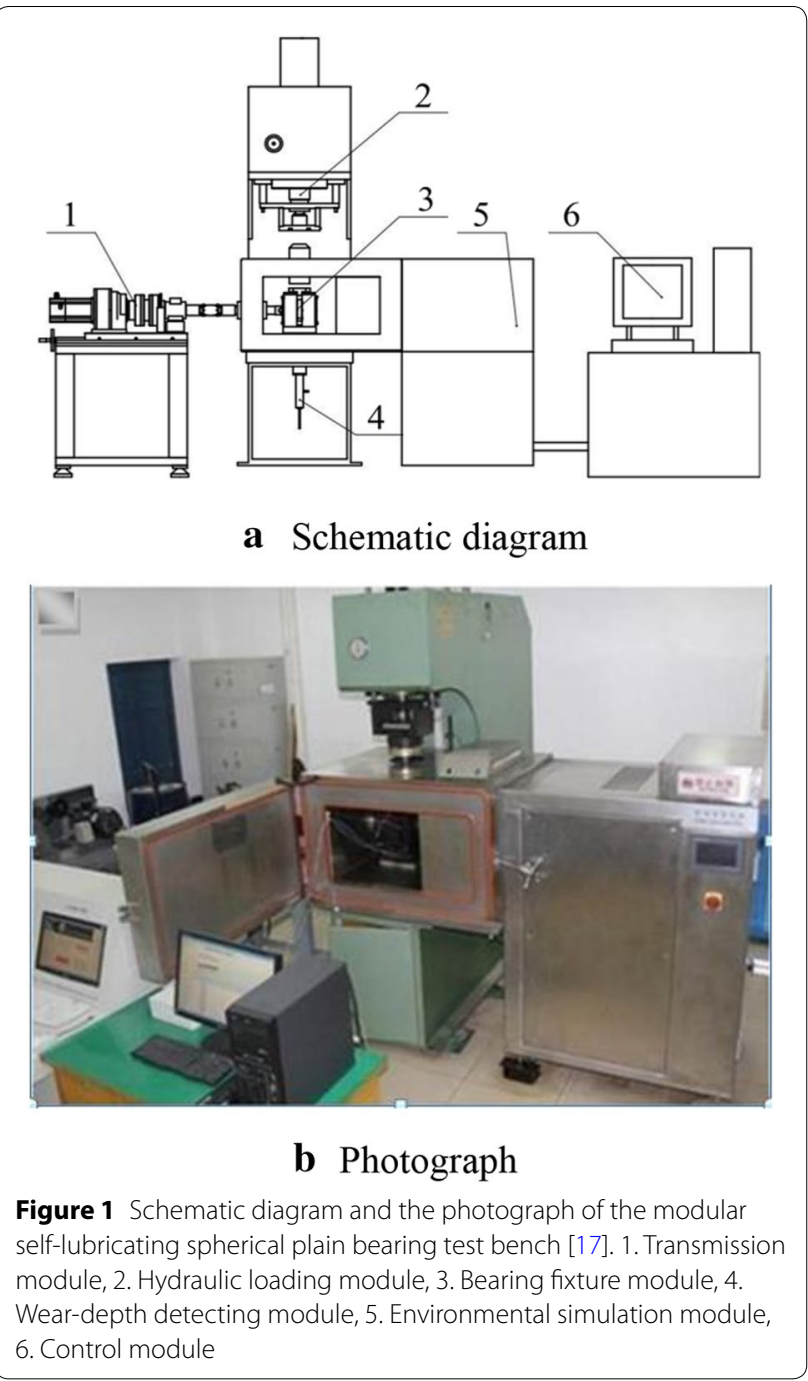




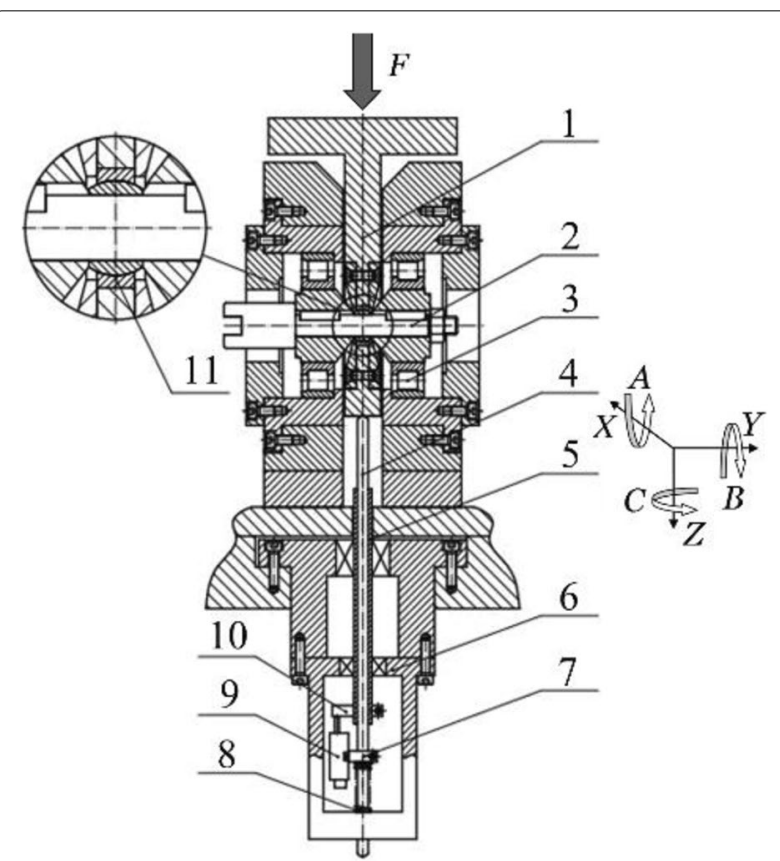

Figure 2 Schematic diagram of the wear-depth detecting system. 1. T-type plate, 2.Testing spindle, 3. Assistant cylindrical roller bearing, 4.Slender pole, 5.Measuring sleeve, 6. Detecting frame, 7. Fixing clamp of displacement sensor, 8. Spring, 9. Displacement sensor, 10. Contacting clamp of displacement sensor, 11. Spherical plain bearing

that the upper end of the slender pole inserted into the measuring sleeve can touch the bottom of the T-type plate by means of the spring force. The displacement sensor (the measuring range is $10 \mathrm{~mm}$, the outstanding linearity is $\pm 0.075 \%$, and the temperature range is from $-30{ }^{\circ} \mathrm{C}$ to $100{ }^{\circ} \mathrm{C}$ ) is fastened on the bottom of the slender pole, and the measuring head of the sensor is pressed onto the contacting clamp of the displacement sensor.

In the wear experiment, the wear of the spherical plain bearing leads to the down-moving of the T-type plate along the $Z$ axis, and the downward displacement is the wear depth of the spherical plain bearing. Meanwhile, the slender pole moves down with the T-type plate along the $Z$ axis, the displacement sensor, fixed on the slender pole, declines along the $Z$ axis and gradually distances the contacting clamp of the displacement sensor (there is a pre-compressing quantity between the displacement sensor and the contacting clamp of the displacement sensor), and at the moment the displacement sensor detects the wear depth of the spherical plain bearing.

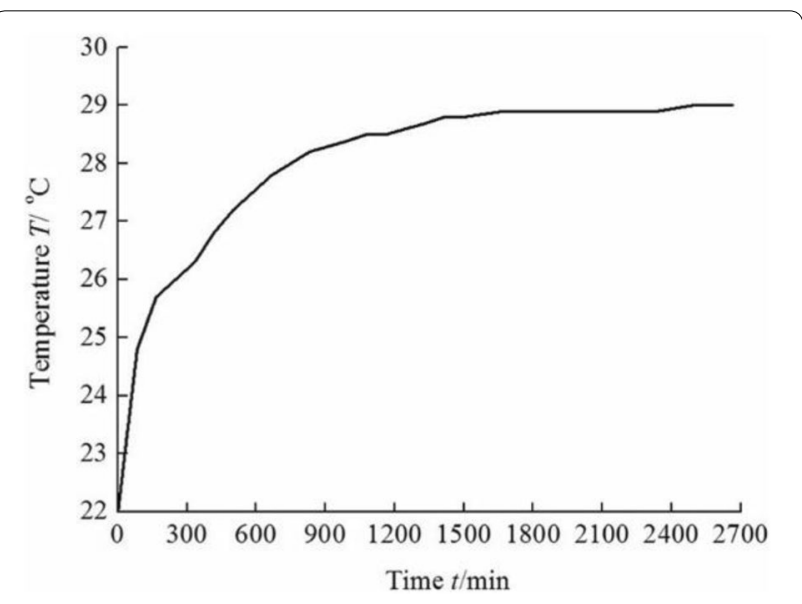

Figure 3 Temperature variation curve of the bearing outer race

\section{Thermal Error Verification of the Wear-Depth Detecting System}

In the wear experiment of spherical plain bearings, there are two main reasons for thermal errors, including the thermal deformation of the wear-depth detecting system caused by the varying environmental temperatures and the thermal deformation caused by the friction heat of bearings (the spherical plain bearing and the assistant rolling bearing) in the bearing fixture module. In the following, the thermal deformation caused by the friction heat is calculated, and the thermal deformation, caused by the environmental temperature variation, is detected in the experiment.

\subsection{Effect of the Friction Heat of Bearings}

On the basis of the low-speed oscillating and heavyloading operating condition (the testing load is $250 \mathrm{MPa}$, the oscillating frequency is $0.2 \mathrm{~Hz}$, and the angle of oscillation is $\pm 25^{\circ}$ ), the thermal deformation of the friction heat of the spherical plain bearing and the temperature rise of the friction heat of the assistant rolling bearing are calculated.

\subsubsection{Effect of the Test Spherical Plain Bearing}

As shown in Figure 2, in the wear experiment process, the testing spindle drives the inner race of the spherical plain bearing to oscillate, and the test loading correspondingly act on the top of the bearing. Then the top of the spherical plain bearing generate friction heat, and then the friction heat of the spherical plain bearing is mainly conducted into the T-type plate by 
Table 1 Required parameters of the thermal deformation simulation of the T-type plate

\begin{tabular}{ll}
\hline Parameters & Value \\
\hline Coefficient of thermal conduction $\lambda(\mathrm{W} /(\mathrm{m} \cdot \mathrm{K}))$ & 50.2 \\
Coefficient of thermal expansion $a(\mu \mathrm{m} / \mathrm{K})$ & 11.59 \\
Air convection coefficient $h\left(\mathrm{~W} /\left(\mathrm{m}^{2} \cdot \mathrm{K}\right)\right)$ & 6 \\
Environmental temperature $\left({ }^{\circ} \mathrm{C}\right)$ & 22 \\
Equilibrium temperature of the outer ring $\left({ }^{\circ} \mathrm{C}\right)$ & 29 \\
\hline
\end{tabular}

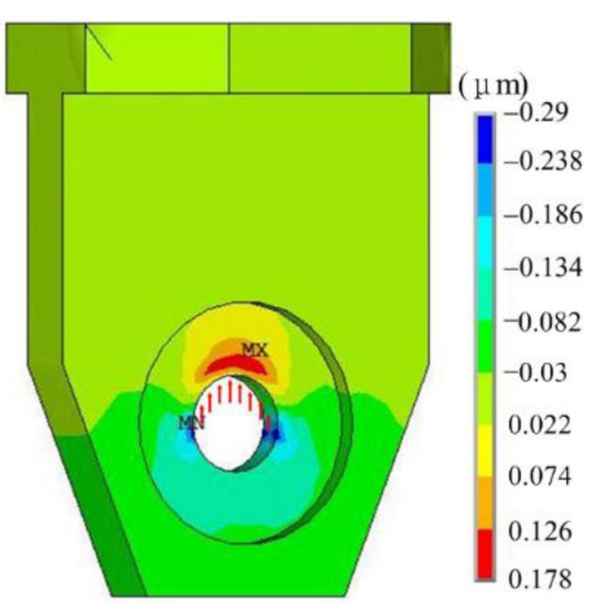

Figure 4 Schematic diagrams of the thermal deformation distribution of the T-type plate

thermal conduction and resulted in the thermal expansion of the T-type plate. In the previous study, the self-lubricating spherical plain bearing reciprocally oscillated 32000 times under the operating condition of low-speed oscillating and heavy-loading, the temperature of the bearing outer race rose about $7{ }^{\circ} \mathrm{C}[18]$. The temperature variation curve of the bearing outer race is shown in Figure 3.

As shown in the Figure 3, the temperature of the outer race of the spherical plain bearing gradually rises from the room temperature $22{ }^{\circ} \mathrm{C}$ to $29^{\circ} \mathrm{C}$ during the experiment. Moreover, the bearing fixture module is in thermal equilibrium, and the temperature of the outer race stays around $29^{\circ} \mathrm{C}$.

At this stage, the thermal deformation of the T-type plate along the $Z$ axis was calculated by FEM after thermal equilibrium of the bearing fixture module. Firstly, the three-dimensional model of the T-type plate was built with Solidworks, and then was simulated with ANSYS. The structural element Solid 186 was adopted to calculate the thermal deformation. The required parameters of this simulation are shown in Table 1.
The schematic diagrams of the thermal deformation is shown in Figure 4. When the bearing fixture module is in thermal equilibrium, the maximum thermal deformation of the T-type plate bottom along the $Z$ axis is $0.074 \mu \mathrm{m}$.

\subsubsection{Effect of the Assistant Rolling Bearing}

As shown in Figure 2, along with the oscillation of the spherical plain bearing, the assistant cylindrical rolling bearing also oscillates in the bearing fixture module, so the assistant rolling bearing generates friction heat under the action of the experimental loading. The heat quantity can be computed by the following equation [19]:

$$
Q=1.047 \times 10^{-4} n M,
$$

where $Q$ is the heat quantity, $n$ is the rotating speed of the bearing, and $M$ is the total frictional torque of the bearing.

The experimental results showed that the rotating speed of the bearing was $12 \mathrm{r} / \mathrm{min}$ and the total frictional torque was $50 \mathrm{Nm}$ [18]. What's more, the friction heat of the rolling bearings were mainly conducted into the bearing fixture (the material of the bearing fixture was $45 \#$, the mass of the bearing fixture was $8.2 \mathrm{~kg}$, and the specific heat capacity was $460 \mathrm{~J} /\left(\mathrm{kg} \cdot{ }^{\circ} \mathrm{C}\right)$ at normal temperature) by thermal conduction. So through the following thermo dynamical formula, the temperature rise was $0.017^{\circ} \mathrm{C}$.

$$
\Delta t=\frac{Q}{C m},
$$

where $\Delta t$ is the temperature rising, $C$ is the specific heat capacity, and $m$ is the mass of the bearing fixture.

From the above calculation, the self-lubricating spherical plain bearing oscillated 32000 times under the operating condition of low-speed oscillating and heavy-loading, the experiment indicated that the temperature of the outer race rose about $7{ }^{\circ} \mathrm{C}$, and the maximum thermal deformation of the T-type plate bottom along the $Z$ axis was $0.074 \mu \mathrm{m}$. The temperature rise of the bearing fixture, caused by the friction heat of the cylindrical rolling bearing, was $0.017^{\circ} \mathrm{C}$.

\subsection{Effect of the Environmental Temperature Variation}

In the experiment, the environmental temperature variation was influenced by the temperature changes in the whole day and the heat dissipation of the embedded hydraulic station. The following experiment was designed to detect the thermal errors of the wear-depth detecting system. 
As shown in Figure 1 and Figure 2, the bearing fixture assembled the self-lubricating spherical plain bearing is placed on the base of the test bench, so that the top of the slender pole tightly touches the bottom of the T-type plate under the spring force. Then the displacement sensor is adjusted and fixed, so that there is a pre-pressing quantity between the displacement sensor and the contacting clamp of the displacement sensor. Without the start-up of the transmission module, there was no relative movement between the inner and outer races of the spherical plain bearing. And when opening embedded hydraulic station, there was no applied load to the spherical plain bearing.

Eight temperature sensors (their sequence numbers were 1-8), collecting the varying temperature of the wear-depth detecting system, were pasted on different locations. The distribution diagram and the photographs of these eight temperature sensors on the wear-depth detecting system are shown in Figure 5.

At the same time, a temperature sensor (No. 9) collecting the room temperature was suspended near the test bench. Another temperature sensor (No. 10), collecting the varying temperature of the hydraulic system, was adhered to the base of the embedded hydraulic station. In conclusion, there were ten temperature sensors on the spherical plain bearing test bench. During the experiment, the corresponding temperature changing curves of the ten temperature sensors are shown in Figure 6, the thermal deformation curve of the wear-depth detecting system along the $Z$ axis is shown in Figure 7 .

As shown in Figure 6 and Figure 7, the total time of the experiment is 525 minute, and the temperature of the ten temperature sensors gradually rises. The temperature variation of the detecting points numbered (1-9) are similar, but the temperature rise of the detecting point (10) on the base of the embedded hydraulic station is higher than the above nine detecting points. The initial indicating value of the displacement sensor is 4.441, and the final value is 4.431 . The thermal deformation of the weardepth detecting system along the $Z$ axis is approximately $0.01 \mathrm{~mm}$ when the room temperature rises $10.3^{\circ} \mathrm{C}$, and the show value of the displacement sensor gradually decreases with the rising of the temperature.

\section{Thermal Errors Modeling}

In view of the above experiment and simulation, the environmental temperature variation is main cause in the two error factors. In order to improve the wear-depth detecting precision, the software compensating method is used to offset the thermal errors of the detecting system. In the following, the compensation model of the thermal errors of the wear-depth detecting system is built.

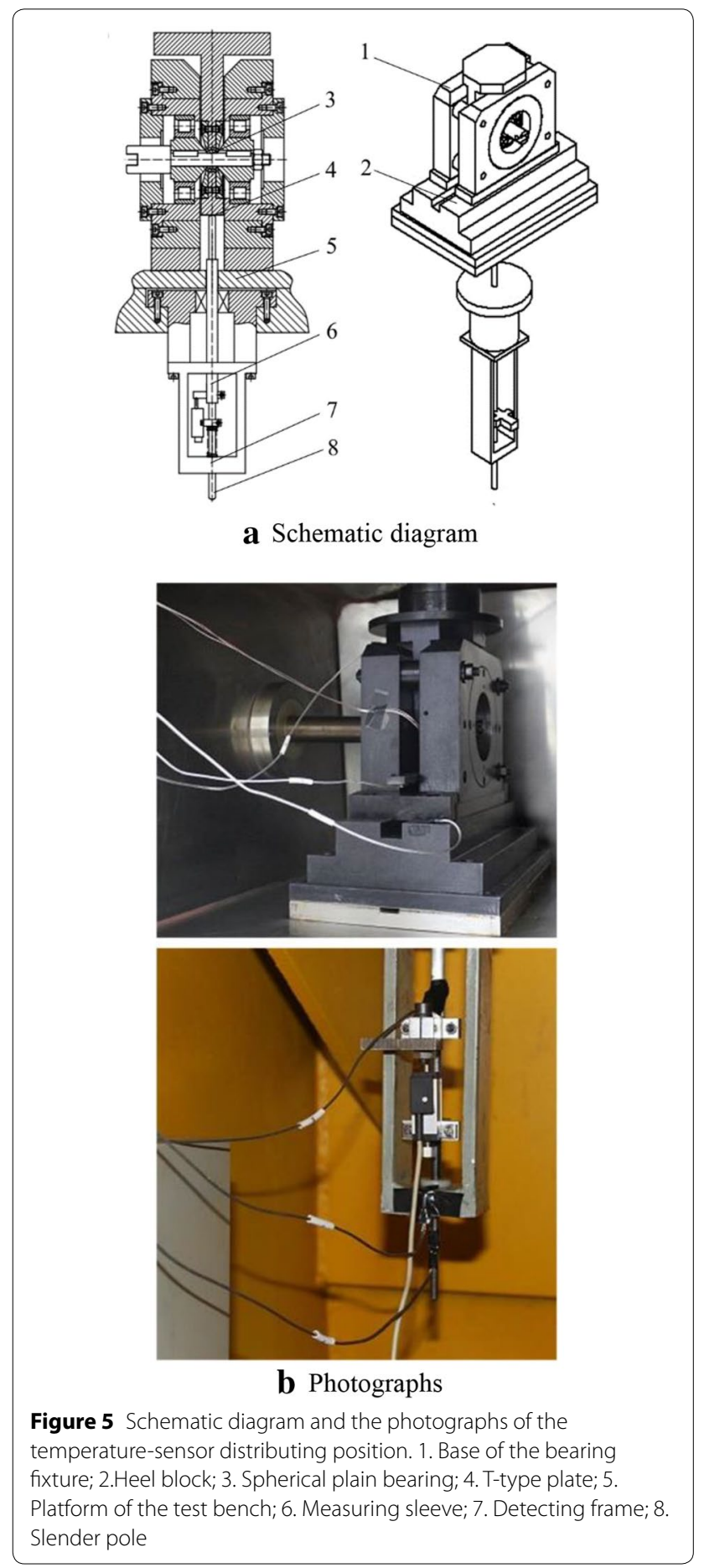

4.1 Selection of the Optimal Temperature Detecting Points In the above ten detecting points, not every point has the same influence on the thermal deformation of the wear-depth detecting system. They may have certain correlations among them, in other words, there is coupling among the ten detecting points. If the mathematical 

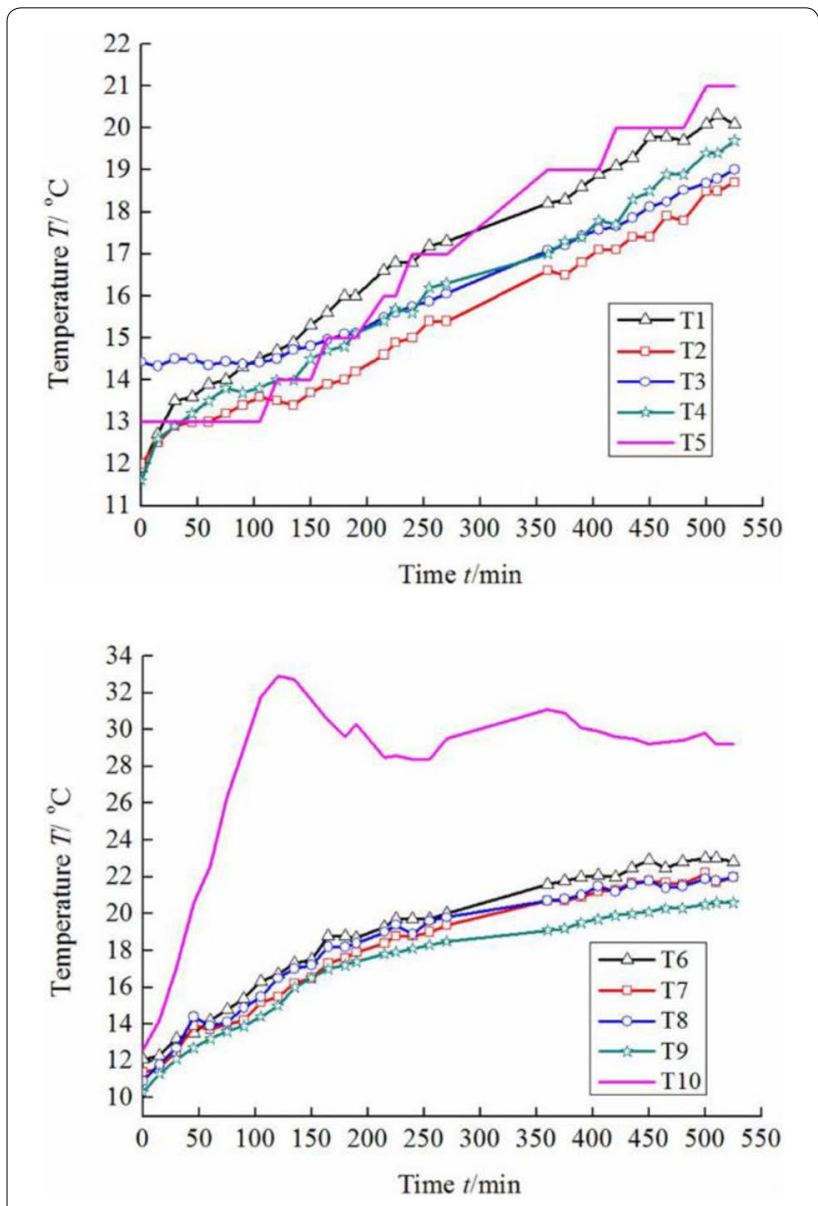

Figure 6 Temperature changing curves of the ten detecting points

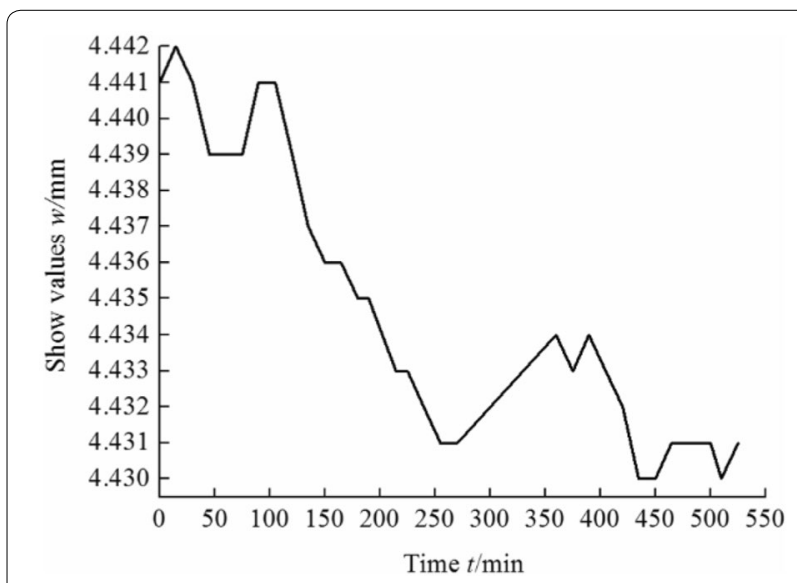

Figure 7 Thermal deformation curve of the wear-depth detecting system along the $Z$ axis

model is directly bulit based on all these detecting-point data, the complexity of the modeling process will be increased while the fitting precision of the model will be reduced. Therefore, in order to get the optimal temperature detecting points for the compensation model of the thermal errors, the ten detecting points on the spherical plain bearing test bench were selected.

In order to estimate the respective influence of the temperature variation of the ten detecting points on the thermal deformation of the detecting system, the correlation coefficients were calculated between the thermal deformation and the temperature changing values of each ten sets. By comparing the correlation coefficients, the most irrelevant detecting point was excluded. Then MATLAB was used to calculate the above correlation coefficients, and the results are listed in Table 2.

From Table 2, the correlation between the temperature variation of the hydraulic station base and the thermal errors is the smallest one, and the value is only 0.537 . Therefore, the temperature changing values of the hydraulic station were excluded in building the thermal error model.

Based on the above analysis, the detecting point of the hydraulic system was excluded. Next, the correlation of the remaining nine detecting points was needed to analyze, and the coupling of the remaining nine detecting points was needed to judge. In this paper, the fuzzy clustering method was used to analyze the mutual correlation of the remaining detecting points by MATLAB, and the remaining nine detecting points were classified on coupling. The results are listed in Table 3.

Table 2 Correlation coefficients between the thermal deformation and the temperature changing values

\begin{tabular}{ll}
\hline Detecting point name & Correlation coefficient \\
\hline 1-Base of the bearing fixture & $\gamma_{1} \rightarrow 0.922$ \\
2-Heel block & $\gamma_{2} \rightarrow 0.871$ \\
3-Spherical plain bearing & $\gamma_{3} \rightarrow 0.841$ \\
4-T-type plate & $\gamma_{4} \rightarrow 0.900$ \\
5-Platform of the test bench & $\gamma_{5} \rightarrow 0.896$ \\
6-Measuring sleeve & $\gamma_{6} \rightarrow 0.927$ \\
7-Detecting frame & $\gamma_{7} \rightarrow 0.940$ \\
8-Slender pole & $\gamma_{8} \rightarrow 0.936$ \\
9-Environmental temperature & $\gamma_{9} \rightarrow 0.943$ (maximum) \\
10-Base of the hydraulic station & $\gamma_{10} \rightarrow 0.537$ (minimum) \\
\hline
\end{tabular}

Table 3 Classification result of the fuzzy clustering method for the remaining nine detecting points

\begin{tabular}{ll}
\hline Category code & $\begin{array}{l}\text { Number } \\
\text { of detecting } \\
\text { points }\end{array}$ \\
\hline First category & 1,3 \\
Second category & 6,8 \\
Third category & $2,4,5$ \\
Fourth category & 7,9 \\
\hline
\end{tabular}


In Table 3, the nine detecting points are divided into four categories. According to the result of the correlation coefficients between the thermal deformation and the temperature changing values of each ten sets, the influence of the temperature rise on the base of the bearing fixture was greater than that on the spherical plain bearing in the first category, namely, $\gamma_{1}>\gamma_{3}$, and the same with the second sort $-\gamma_{8}>\gamma_{6}$, the third sort $-\gamma_{4}>\gamma_{5}>\gamma_{2}$, and the fourth sort- $\gamma_{9}>\gamma_{7}$. Therefore, the greatest influencing detecting points were selected in the above four categories: in the first category-(1) the base of the bearing fixture, in the second one-(8) the slender pole, in the third-(4) the T-type plate, and in the fourth-(9) the environmental temperature.

\subsection{Modeling Process of the Thermal Error Compensation} In selecting the four optimal detecting points, MLR and time series analysis were adopted to build the comprehensive compensating model of the wear-depth detecting system in the following.

\subsubsection{Multiple Linear Regression Modeling}

MATLAB was used to fit the multiple linear model of the four optimal detecting points and the thermal deformation of the detecting system, as follows:

$$
\begin{aligned}
y_{M}(t)= & 4.456-0.0015 \Delta T_{1}(t)+0.0008 \Delta T_{4}(t) \\
& +0.0006 \Delta T_{8}(t)-0.0012 \Delta T_{9}(t),
\end{aligned}
$$

where $y_{M}(t)$ is the fitting values of the multiple linear model, $\Delta T_{1}(t)$ is the temperature variation of the base of the bearing fixture, $\Delta T_{4}(t)$ is the temperature variation of the T-type plate, $\Delta T_{8}(t)$ is the temperature variation of the slender pole, and $\Delta T_{9}(t)$ is the temperature variation of the environment.

Hence, the curves of the multi-element fitting thermal errors and the detecting thermal errors are shown in Figure 8, the residual curve of the multi-element fitting errors and the detecting thermal errors is shown in Figure 9.

As shown in Figure 8 and Figure 9, the curve similarity between the multi-element fitting thermal errors and the detecting thermal errors is low, and the maximum residual error between the multi-element fitting value and the real detecting value is approximately $2 \mu \mathrm{m}$.

On the basis of the above fitting results, there are residual errors $\delta(t)$ between the multi-element fitting value $y_{M}(t)$ and the real detecting value $y_{D}(t)$, and the relationship among them is shown below:

$$
y_{D}(t)=y_{M}(t)+\delta(t),
$$

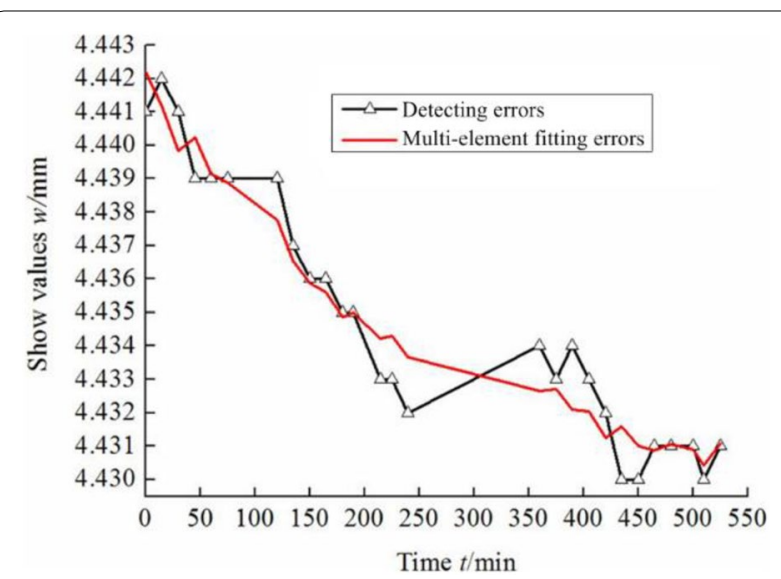

Figure 8 Curves of the multi-element fitting thermal errors and the detecting thermal errors

where $y_{D}(t)$ is the real detecting value, and $\delta(t)$ is the residual errors of the multi-element fitting value. From Eqs. (3) and (4), $y_{D}(t)$ can be gotten:

$$
\begin{aligned}
y_{D}(t)= & 4.456-0.0015 \Delta T_{1}(t)+0.0008 \Delta T_{4}(t) \\
& +0.0006 \Delta T_{8}(t)-0.0012 \Delta T_{9}(t)+\delta(t),
\end{aligned}
$$

In order to further improve the fitting precision of the thermal error model of the wear-depth detecting system, on the basis of multi-element fitting, time series analysis method was introduced for the residual errors $\delta(t)$, which improved the curve similarity between the multi-element fitting thermal errors and the detected thermal errors. The time series analysis, processing the dynamic data, is a parameterized analytical method, and it could fit the applicable time series model on the basis of the random data arranged according to the time sequence. The above model was used to analyze the data system to learn the inner structure and the dynamic property of the random data. Thus the data trend could be predict with the existing data [20].

The data stationarity is the basis of time series analysis, so the stationarity of the multi-element fitting residual errors $\delta(t)$ should be verified first.

The following is the stationarity verifying of the multi-element fitting residual errors $\delta(t)$. The method used in following formulas is inverse number [20]:

$$
\left\{\begin{array}{l}
E A=\frac{1}{4} N(N-1) \\
D A=\frac{1}{72}\left(2 N^{3}+3 N^{2}-5 N\right),
\end{array}\right.
$$




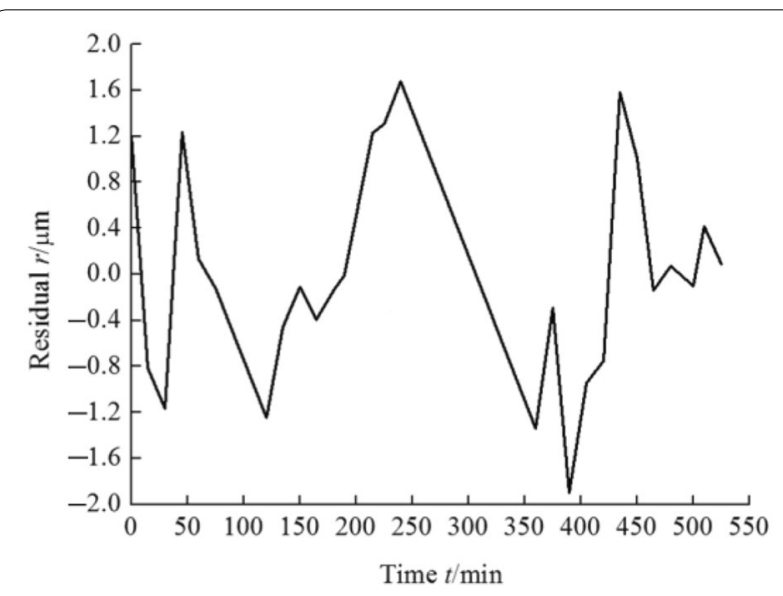

Figure 9 Residual curve of the multi-element fitting errors and the detected errors

$$
u=\frac{A+\frac{1}{2}-E A}{\sqrt{D A}}
$$

where $A$ is the inverse number of the residual errors $\delta(t)$, and $N$ is the number of values.

In view of the given $\alpha=0.05$, if $|u|<u_{\alpha / 2}$, the multielement fitting residual errors $\delta(t)$ is stable and vice versa.

In Eqs. (6) and (7), $N=27$ and $A=378$. So $u=0.1764<u_{\alpha / 2}=1.96$. The multi-element fitting residual errors $\delta(t)$ is stable.

The normal distribution testing of the multi-element fitting residual errors $\delta(t)$ is done as follows.

The white noise with the normal distribution characteristics is the premise of the time series model, that is, the multi-element fitting residual errors $\delta(t)$ should obey the normal distribution. The existing function of the normal distribution test in MATLAB was used to get that the multi-element fitting residual errors $\delta(t)$ obeyed the normal distribution.

\subsubsection{Model Selection of Time Series Analysis}

From the above stationarity verifying and normal distribution testing, the multi-element fitting residual errors
Table 4 Selection criterions of the model type [22]

\begin{tabular}{lll}
\hline Model type & ACF & PACF \\
\hline $\operatorname{AR}(p)$ & Trail off & Cut off \\
$\operatorname{MA}(q)$ & Cut off & Trail off \\
$\operatorname{ARMA}(p, q)$ & Trail off & Trail off \\
\hline
\end{tabular}

$\delta(t)$ met the conditions of time series analysis. At present, there are three common models for time series analysis, namely, autoregression model-AR $(p)$, moving average model-MA $(q)$ and autoregressive moving average model- $\operatorname{ARMA}(p, q)[21]$. At the beginning, a model type of the time series modeling need to be determined based on the above three common models. Meanwhile, the features of the two function values, autocorrelation function (ACF) and partial autocorrelation function (PACF), affect the selection of the model type. The relationship between the features of two function values and the model type are shown in Table 4.

The MATLAB was used to calculate autocorrelation function (ACF) and partial autocorrelation function (PACF) of the multi-element fitting residual errors $\delta(t)$. The function values are shown in Figure 10.

As shown in Figure 10, the features of autocorrelation function (ACF) and partial autocorrelation function (PACF) of the multi-element fitting residual errors $\delta(t)$ are both "trail off". According to Table 4, the model type is the autoregressive moving average model- $\operatorname{ARMA}(p, q)$.

\subsubsection{Model Order Determination and Fitting}

As mentioned above, the model type was selected as $\operatorname{ARMA}(p, q)$, and then the model order determination, namely the number of " $p$ " and " $q$ ", should be determined.

The order determination needed to meet three criteria-the AIC criterion, the FPE criterion and the white noise criterion [20]. To start with, the function of time series analysis, the function of the AIC criterion and the function of the FPE criterion in MATLAB were used to primarily determine the model order as $\operatorname{ARMA}(6,4)$. The time series fitting residual errors $\delta(t)^{\prime}$ was then got and the corresponding formula was shown as follows:

$$
\begin{aligned}
\delta(t)^{\prime}= & 0.1821 \delta(t-1)^{\prime}+0.7783 \delta(t-2)^{\prime}-0.9575 \delta(t-3)^{\prime}- \\
& 0.1277 \delta(t-4)^{\prime}+0.2992 \delta(t-5)^{\prime}-0.4679 \delta(t-6)^{\prime}+ \\
& \varepsilon(t)-0.03235 \varepsilon(t-1)-1.781 \varepsilon(t-2)+0.02422 \varepsilon(t-3)+ \\
& 0.8183 \varepsilon(t-4),
\end{aligned}
$$




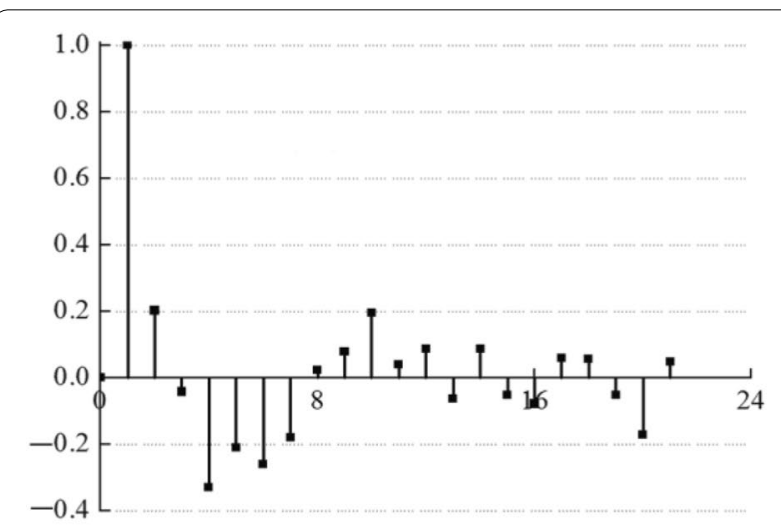

a $\mathrm{ACF}$

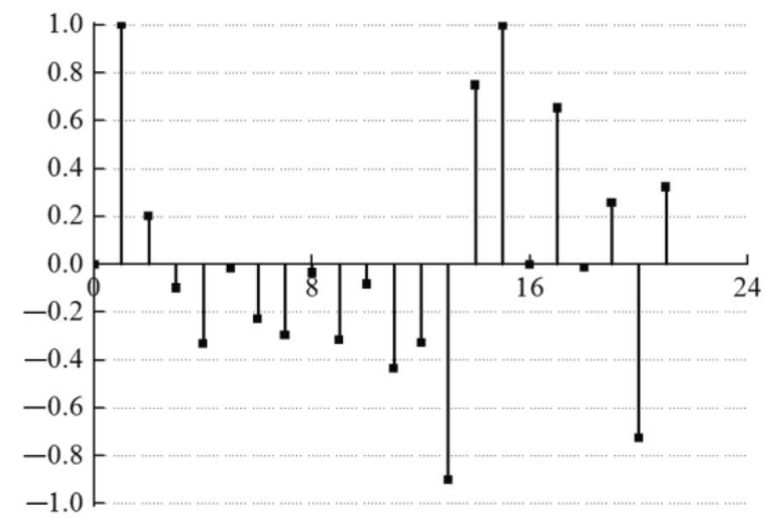

b PACF

Figure $10 \mathrm{ACF}$ and PACF of the multi-element fitting residual errors

where $\delta(t)^{\prime}$ is the residual errors of the time series fitting, and $\varepsilon(t)$ is the unknown and unpredictable residual errors.

\subsubsection{Equivalent Long Auto-regressive Algorithm}

In Eq. (8), the residual errors $\varepsilon(t)$ were unknown in the model ARMA $(6,4)$, so the time series fitting residual

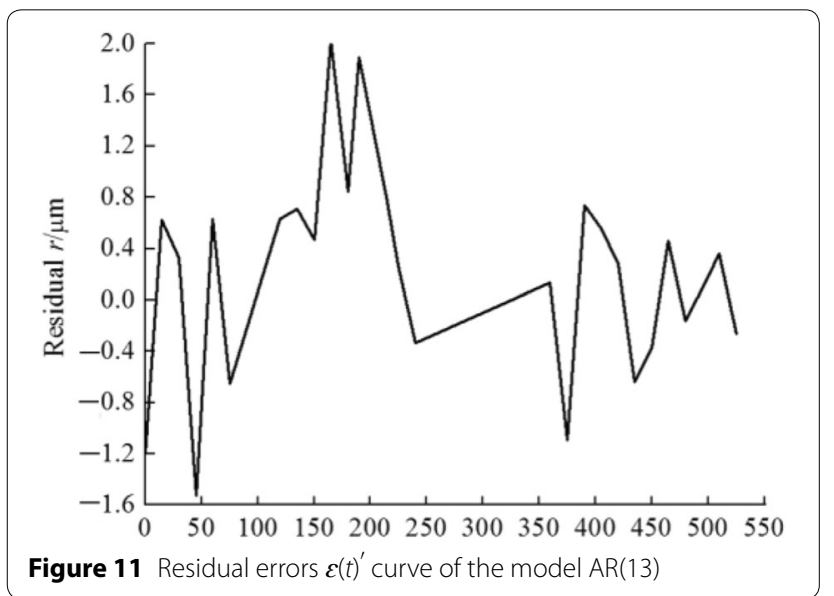

$\operatorname{ARMA}(p, q)$. And the residual errors $\varepsilon(t)^{\prime}$ of the model $\mathrm{AR}(w)$ could also replace the unknown residual errors $\varepsilon(t)$ in the model ARMA $(6,4)$.

In the following, the equivalent long auto-regressive algorithm was used to fit model $\mathrm{AR}(w)$ for the multi-element fitting residual errors $\delta(t)$, and the model order $w$ was calculated by the below formula [21]:

$$
w=(2 \sim 5) N^{0.3},
$$

where $w$ is the order of the model $\operatorname{AR}(w)$.

After the model order was determined, the model $\mathrm{AR}(w)$ of the multi-element fitting residual errors $\delta(t)$ was fitted with MATLAB:

$$
\delta(t)^{\prime \prime}=\sum_{i=1}^{w}-\Phi_{i} \delta(t-i)^{\prime \prime}+\varepsilon(t)^{\prime},
$$

where $\varepsilon(t)^{\prime}$ is the residual errors of the model $\operatorname{AR}(w)$, and $\delta(t)^{\prime \prime}$ is the fitting values of the model $\mathrm{AR}(w)$.

In Eq. (9), $N=27$, and then $w=13$. So the high-order auto-regressive model was $\mathrm{AR}(13)$, and the formula of the model AR(13) was shown as below:

$$
\begin{aligned}
\delta(t)^{\prime \prime}= & -0.1555 \delta(t-1)^{\prime \prime}-0.8027 \delta(t-2)^{\prime \prime}-0.9294 \delta(t-3)^{\prime \prime} \\
& -0.6108 \delta(t-4)^{\prime \prime}-1.122 \delta(t-5)^{\prime \prime}-0.832 \delta(t-6)^{\prime \prime} \\
& -0.9551 \delta(t-7)^{\prime \prime}-0.9243 \delta(t-8)^{\prime \prime}-0.5616 \delta(t-9)^{\prime \prime} \\
& -0.8522 \delta(t-10)^{\prime \prime}-0.3505 \delta(t-11)^{\prime \prime}-0.5723 \delta(t-12)^{\prime \prime} \\
& -0.147 \delta(t-13)^{\prime \prime}+\varepsilon(t)^{\prime},
\end{aligned}
$$

errors $\delta(t)^{\prime}$ were not calculated by the linear derivation. However, if the model order $w$ was large enough, the model $\mathrm{AR}(w)$ could approximately substitute the model
From the above, Eqs. (5) and (11), the residual errors $\varepsilon(t)^{\prime}$ of the $\operatorname{AR}(13)$ was got. The residual errors $\varepsilon(t)^{\prime}$ curve is shown in Figure 11. 


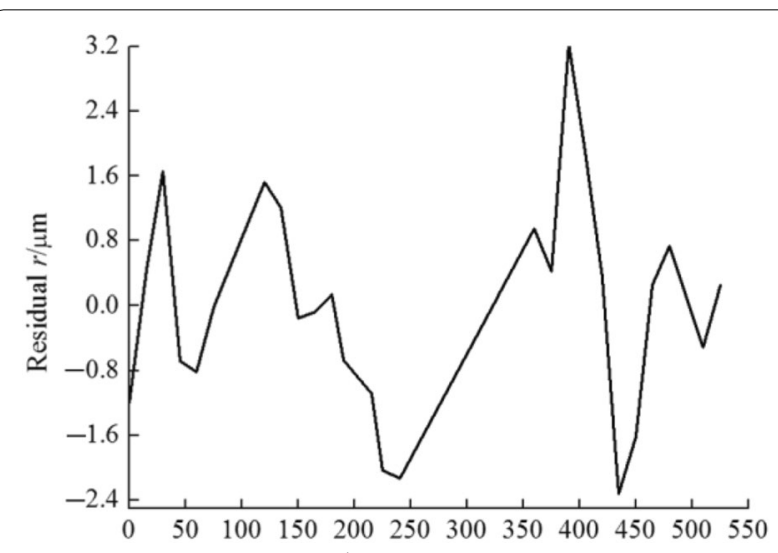

Figure 12 Residual errors $\delta(t)^{\prime}$ curve of the time series analysis

The white noise verification of the residual errors $\varepsilon(t)^{\prime}$ of the model AR(13).

After calculating the residual errors $\varepsilon(t)^{\prime}$ of the model $\operatorname{AR}(13)$, the residual errors $\varepsilon(t)^{\prime}$ needed to be verified whether they were the white noise. Finally, the test function of the white noise in MATLAB was used and the residual errors $\varepsilon(t)^{\prime}$ of the model AR(13) were verified to be the white noise. Thus the residual errors $\varepsilon(t)^{\prime}$ of the model $\mathrm{AR}(13)$ could replace the residual errors $\varepsilon(t)$ of the model ARMA $(6,4)$.

\subsubsection{Residual Errors $\delta(t)^{\prime}$ Fitting of Time Series Analysis}

Since the residual errors $\varepsilon(t)^{\prime}$ of the model AR(13) could replace the residual errors $\varepsilon(t)$ of the model $\operatorname{ARMA}(6,4)$, according to Eq. (8), Eq. (12) was got:

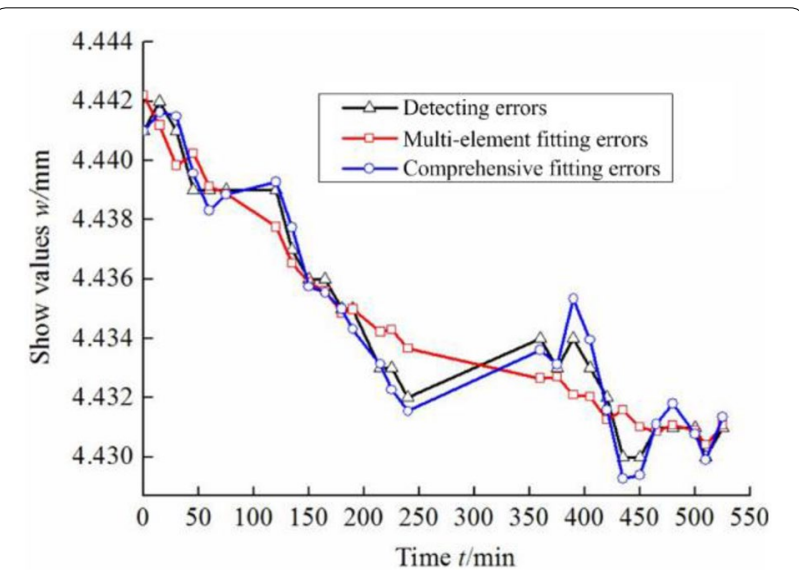

Figure 13 Comparisons of three thermal error curves

residual errors $\delta(t)^{\prime}$ of the time series analysis and the fitting value of the multiple linear model $y_{M}(t)$, the comprehensive compensation model $y_{C}(t)$ of the thermal errors of the wear-depth detecting system was obtained.

\subsubsection{Comprehensive Compensation Model of the Thermal Errors}

Combining with the multi-element fitting value $y_{M}(t)$, as shown in Eq. (3), the comprehensive compensation model of the thermal errors of the wear-depth detecting system $y_{C}(t)$ was got:

$$
y_{C}(t)=y_{M}(t)+\delta(t)^{\prime}
$$

where $y_{C}(t)$ is the fitting values of the comprehensive compensation model.

$$
\begin{aligned}
\delta(t)^{\prime}= & 0.1821 \delta(t-1)^{\prime}+0.7783 \delta(t-2)^{\prime}-0.9575 \delta(t-3)^{\prime} \\
& -0.1277 \delta(t-4)^{\prime}+0.2992 \delta(t-5)^{\prime}-0.4679 \delta(t-6)^{\prime} \\
& +\varepsilon(t)^{\prime}-0.03235 \varepsilon(t-1)^{\prime}-1.781 \varepsilon(t-2)^{\prime} \\
& +0.02422 \varepsilon(t-3)^{\prime}+0.8183 \varepsilon(t-4)^{\prime},
\end{aligned}
$$

So the residual errors $\delta(t)^{\prime}$ of the time series analysis (this was also the residual errors of the comprehensive compensation model) for the thermal errors were calculated. The residual errors $\delta(t)^{\prime}$ curve is shown in Figure 12.

The white noise verification of the residual errors $\delta(t)^{\prime}$ of time series analysis.

After the residual errors $\delta(t)^{\prime}$ of time series analysis in Eq. (12) were calculated, the residual errors $\delta(t)^{\prime}$ needed to be verified whether they were the white noise. Finally, the test function of the white noise in MATLAB was used to verify that the residual errors $\delta(t)^{\prime}$ of the time series analysis were the white noise. Thus, combining with the
The real detecting thermal error curve of the weardepth detecting system, the thermal error curve of the multi-element model fitting and the thermal error curve of the comprehensive compensation model identification are shown in Figure 13, and the residual curves of the multi-element model fitting and the comprehensive compensation model identification are shown in Figure 14.

As shown in Figure 13 and Figure 14, compared with the curve of the multi-element model fitting, the curve of the comprehensive compensation model identification is more similar to the real detected thermal errors curve. In conclusion, the residual errors of the comprehensive compensation model identification are less than 




Figure 14 Residual curves of the multi-element model fitting and the comprehensive compensation model identification

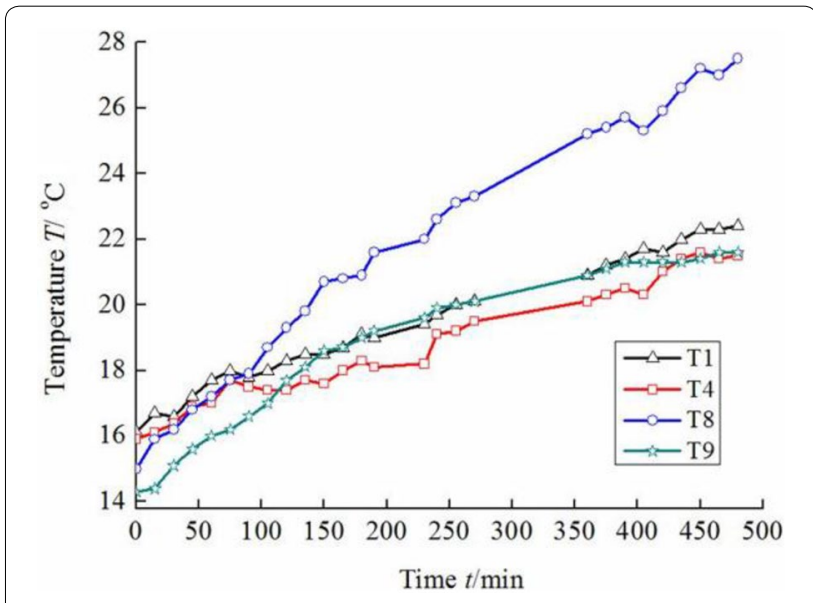

Figure 15 Temperature curves of the four optimal detecting points

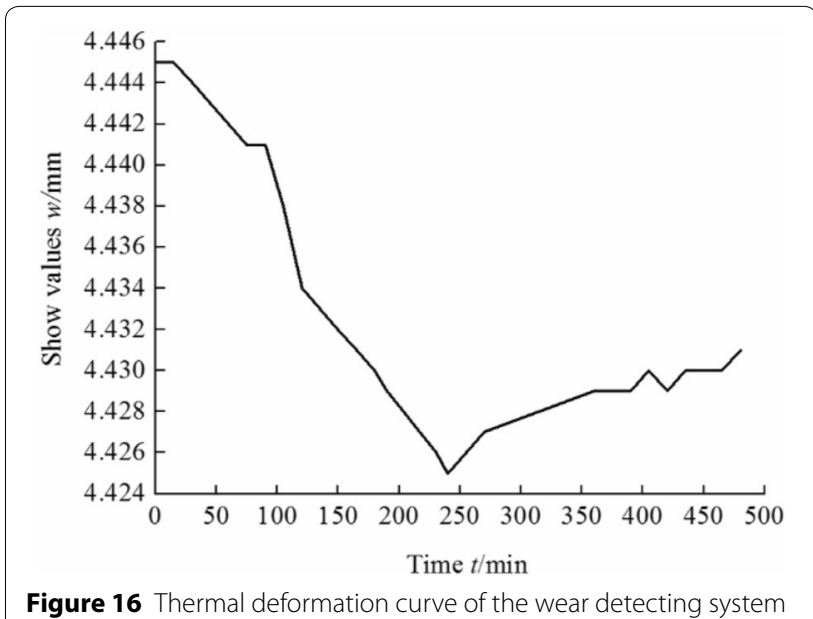

that of the multi-element model fitting except for very few points. The maximum residual errors between the multi-element model fitting and the real detecting thermal errors is $2 \mu \mathrm{m}$, and the maximum residual between the comprehensive compensation model identification and the real detected thermal errors is $1 \mu \mathrm{m}$.

\section{Applicability Verification of the Comprehensive Compensation Model of Thermal Errors}

As mentioned above, the comprehensive compensation model had higher fitting precision. Then in order to verify the applicability of this compensation model, this comprehensive compensation model was used to fit other thermal experimental data.

As the selection of the optimal temperature detecting points (in Section 4.1) showed, this experiment only collected the temperature changing values of the four detecting points, namely, (1) the base of the bearing fixture, (4) the T-type plate, (8) the slender pole, and (9) the environmental temperature. In the thermal error experiment, the corresponding temperature changing curves of the four optimal temperature detecting points are shown in Figure 15; the thermal deformation curve of the weardepth detecting system is shown in Figure 16.

As shown in Figure 15 and Figure 16, the total time of the experiment is $480 \mathrm{~min}$, and the temperature of the four optimal temperature points gradually rises. The initial indicating value of the displacement sensor is 4.445 , and the final value is 4.431 . The maximum thermal error of the wear-depth detecting system is $0.02 \mathrm{~mm}$.

The comprehensive compensation model identification: we put the four temperature varying data into the multiple linear model (Eq. (3)), and got the multiple linear fitting values. Then we introduced the time series analysis method and fitted the residual errors of the multiple linear model. At last, we got the comprehensive compensation model. The curves of the comprehensive compensation model identification and the detecting thermal errors are shown in Figure 17; the residual error curve of the comprehensive compensation model and the detecting errors is shown in Figure 18.

As shown in Figure 17 and Figure 18, the comprehensive compensation model curve is similar to the detecting thermal error curve, and the maximum residual error is about $9 \mu \mathrm{m}$. The maximum thermal error of the weardepth detecting system is $0.02 \mathrm{~mm}$, and the comprehensive compensation model could offset the thermal errors about $0.01 \mathrm{~mm}$. Depending on the above verification, the comprehensive compensation model, built through MLR and time series analysis method, had the higher fitting precision and better applicability. 


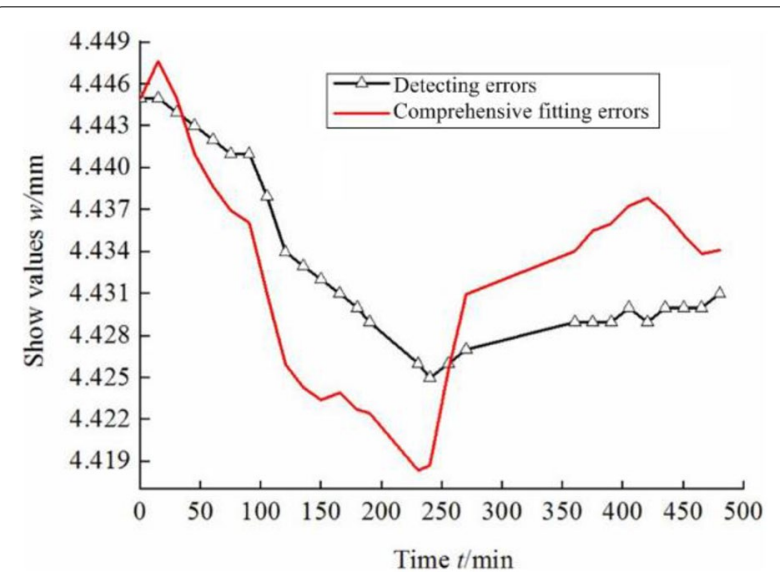

Figure 17 Curves of the comprehensive compensation model identification values and the detecting thermal errors

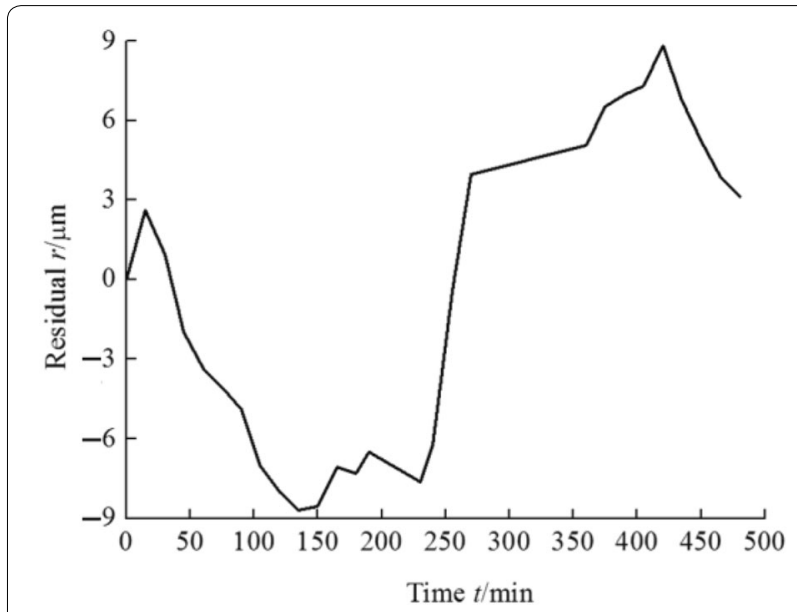

Figure 18 Residual curve of the comprehensive compensation model identification values and the detecting errors

\section{Conclusions}

(1) Under the operating condition of the low-speed oscillating and heavy-loading, the thermal deformation of the T-type plate caused by the friction heat of spherical plain bearings is $0.074 \mu \mathrm{m}$ when the spherical plain bearing oscillates 32000 times and the bearing outer race temperature rises about $7{ }^{\circ} \mathrm{C}$, and the bearing fixture temperature due to the friction heat of cylindrical roller bearings rises $0.017^{\circ} \mathrm{C}$

(2) The thermal deformation of the wear-depth detecting system is approximately $0.01 \mathrm{~mm}$ when the room temperature rises $10.3{ }^{\circ} \mathrm{C}$, and the showing value of the displacement sensor gradually decreases with the rising of the temperature. In view of the above results, the environmental temperature variation is the main cause of the two error factors.

(3) The comprehensive compensating model of the thermal error of the wear-depth detecting system is built with MLR and time series analysis. The fitting curve of the comprehensive compensation model is more similar to the real detecting curve of the thermal errors, and the maximum residual of the two curves is only $1 \mu \mathrm{m}$. Finally, according to the experimental verification, the comprehensive compensation model has higher fitting precision and better applicability.

\section{Authors' Contributions}

Z-QH was in charge of the whole trial; WL wrote the manuscript; Y-LY, B-LF, and $\mathrm{H}-\mathrm{LZ}$ assisted with sampling and laboratory analyses. All authors read and approved the final manuscript.

\section{Authors' Information}

Zhan-Qi Hu, born in 1956, is currently a professor and a PhD candidate supervisor at Aviation Key Laboratory of Science and Technology on Generic Technology of Aviation Self-Lubricating, Yanshan University, China. His main research interests include CNC technology and intelligent manufacturing.

Wei Li, born in 1985, is currently a PhD candidate at Aviation Key Laboratory of Science and Technology on Generic Technology of Aviation Self-Lubricating, Yanshan University, China. He received his master degree from Yanshan University, China, in 2012. His research interests include error compensation and detecting credibility for the tribological testers.

Yu-Lin Yang, born in 1953, is currently a professor and a PhD candidate supervisor at Aviation Key Laboratory of Science and Technology on Generic Technology of Aviation Self-Lubricating, Yanshan University, China. His main research interests include nano-tribology and self-lubricating bearings.

Bing-Li Fan, born in 1982, is currently a research fellow at Aviation Key Laboratory of Science and Technology on Generic Technology of Aviation SelfLubricating, Yanshan University, China. His main research interests include self-lubricating fabric liners and bearings.

Hai-Li Zhou, born in 1989, is currently a PhD candidate at Aviation Key Laboratory of Science and Technology on Generic Technology of Aviation Self-Lubricating, Yanshan University, China. She received her bachelor degree from Yanshan University, China, in 2012. Her main research interests include mechanical property of the expanded graphite.

\section{Competing Interests}

The authors declare no competing financial interests.

\section{Funding}

Supported by National Natural Science Foundation of China (Grant No. 51405422), Hebei Provincial Natural Science Foundation of China (Grant No. E2015203113), and Technological Innovation Fund of Aviation Industry of China (Grant No. 2014E00468R).

\section{Publisher's Note}

Springer Nature remains neutral with regard to jurisdictional claims in published maps and institutional affiliations.

Received: 18 March 2016 Accepted: 27 September 2018 Published online: 12 October 2018 


\section{References}

[1] XW Qi, J Ma, Z N Jia, et al. Effects of weft density on the friction and wear properties of self-lubricating fabric for journal bearings under heavy load conditions. Wear, 2014, 318(1): 124-129.

[2] Y Hu, Y T Fei, W T Cheng. Thermal deformation error and correction for articulated arm coordinate-measuring machines. Journal of Mechanical Engineering, 2011, 47(24): 15-19. (in Chinese)

[3] M Weck, P Mckeown, R Bonse, et al. Reduction and compensation of thermal errors in machine tools. CIRP Annals_-Manufacturing Technology, 1995, 44(2): 589-598.

[4] K Zhou. Design for the test machine of spherical plain bearing and the accuracy compensation. Qinhuangdao: Yanshan University, 2012. (in Chinese)

[5] Z Q Hu, W Li, Y L Yang, et al. The high and low temperature environment test bench of spherical plain bearings: China, CN103048136A. 2013-417[2016-8-16]. http://d.g.wanfangdata.com.cn/Patent_CN20121055 6215.2.aspx.

[6] W Li, Z Q Hu, Y L Yang, et al. Comprehensive error modeling of real-time wear-depth detecting of spherical plain bearing tester. Optics and Precision Engineering, 2016, 24(4): 844-854. (in Chinese)

[7] Y Hong, N Jun. Dynamic neural network modeling for nonlinear, nonstationary machine tool thermally induced error. International Journal of Machine Tools \& Manufacture, 2005, 45(4): 455-465.

[8] J Han, L P Wang, HTWang, et al. A new thermal error modeling method for CNC machine tools. International Journal of Advanced Manufacturing Technology, 2011, 62(1-4): 205-212.

[9] W L Feng, Z H Li, Q Y Gu. Thermally induced positioning error modelling and compensation based on thermal characteristic analysis. International Journal of Machine Tools \& Manufacture, 2015, 93: 26-36.

[10] T Reddy, V Shanmugaraj, V Prakash, et al. Real-time thermal error compensation module for intelligent ultra precision turning machine. Procedia Materials Science, 2014, 6: 1981-1988.

[11] B Tan, XY Mao, H Q Liu. A thermal error model for large machine tools that considers environmental thermal hysteresis effects. International Journal of Machine Tools \& Manufacture, 2014, 82-83(7): 11-20.
[12] A M Abdulshahed, A P Longstaff, S Fletcher. The application of ANFIS prediction models for thermal error compensation on CNC machine tools. Applied Soft Computing, 2015, 27(c): 158-168.

[13] A M Abdulshahed, A P Longstaff, S Fletcher. Thermal error modelling of machine tools based on ANFIS with fuzzy c-means clustering using a thermal imaging camera. Applied Mathematical Modelling, 2014, 39(7): 1837-1852.

[14] R Ramesh, M A Mannan, A N Poo, et al. Thermal error measurement and modelling in machine tools. Part II. hybrid Bayesian network-support vector machine model. International Journal of Machine Tools \& Manufacture, 2003, 43(4): 405-419.

[15] H Wu, HT Zhang, Q J Guo. Thermal error optimization modeling and real-time compensation on a CNC turning center. Journal of Materials Processing Technology, 2008, 207(1-3): 172-179.

[16] Q J Guo, J G Yang, H Wu. Application of ACO-BPN to thermal error modeling of NC machine tool. International Journal of Advanced Manufacturing Technology, 2010, 50(5): 667-675.

[17] W Li, Z Q Hu Z, Y L Yang, et al. Modeling and verification of comprehensive errors of real-time wear-depth detecting for spherical plan bearing tester. Journal of Central South University, 2018, 3(3): 533-545.

[18] D Zhang. Frictional heat temperature field simulation and test of selflubricating spherical plain bearing. Qinhuangdao: Yanshan University, 2012. (in Chinese)

[19] TY Chen, W J Wei, J C Tsai. Optimum design of headstocks of precision lathes. International Journal of Machine Tools \& Manufacture, 1999, 39(12): 1961-1977.

[20] S Z Yang, Y Wu, J P Xuan. Time series analysis in engineering application. Wuhan: Huazhong University of Science and Technology Press, 2004. (in Chinese)

[21] H H Lin. Data processing of dynamic measurement. Beijing: Beijing Institute of Technology Press, 1995. (in Chinese)

[22] Z G Liu, Z J Cai, X M Tan. Forecasting research of aero-engine rotate speed signal based on arma model. Procedia Engineering, 2011, 15: 115-121.

\section{Submit your manuscript to a SpringerOpen ${ }^{\circ}$ journal and benefit from:}

- Convenient online submission

- Rigorous peer review

- Open access: articles freely available online

- High visibility within the field

Retaining the copyright to your article

Submit your next manuscript at springeropen.com 